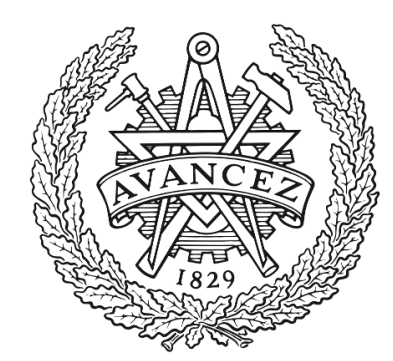

\title{
CHALMERS
}

UNIVERSITY OF TECHNOLOGY

\section{Robust Connectivity With Multiple Directional Antennas for Vehicular Communications}

Downloaded from: https://research.chalmers.se, 2023-04-26 10:37 UTC

Citation for the original published paper (version of record):

Nagalapur, K., Ström, E., Brännström, F. et al (2020). Robust Connectivity With Multiple Directional Antennas for Vehicular Communications. IEEE Transactions on Intelligent Transportation Systems, 21(12): 5305-5315. http://dx.doi.org/10.1109/TITS.2019.2956099

N.B. When citing this work, cite the original published paper.

(C2020 IEEE. Personal use of this material is permitted.

However, permission to reprint/republish this material for advertising or promotional purposes 


\title{
Robust Connectivity with Multiple Directional Antennas for Vehicular Communications
}

\author{
Keerthi Kumar Nagalapur, Erik G. Ström, Fredrik Brännström, Jan Carlsson, and Kristian Karlsson
}

\begin{abstract}
For critical vehicular communication services, such as traffic safety and traffic efficiency, it is advisable to design systems with robustness as the main criteria, possibly at the price of reduced peak performance and efficiency. We describe a simple, low-cost method for combining the output of $L$ directional (i.e., not omnidirectional) antennas to the input of a single-port receiver with the aim to guarantee robustness, i.e., to minimize the probability that $K$ consecutive packets arriving from the worst-case angle-of-arrival are decoded incorrectly. To minimize complexity, the combining network does not estimate or use channel state information. The combining network consists of $L-1$ analog phase shifters whose phases are affine functions of time. For a general $L \leq K$ and when the packet error probability decays exponentially with the received SNR, the optimum slopes of the affine functions can be computed by solving an optimization problem that depends on the antenna far-field functions. We provide analytical solutions for the special case of $L=2$ and 3 antennas, which turns out to be independent of the antenna far-field functions and placement on a vehicle. In an experimental setup consisting of two monopole antennas mounted on the roof of a Volvo XC90, the proposed combining method is shown to give significant performance gains, compared to using any one of the antennas.
\end{abstract}

Index Terms-Robustness, vehicular communications, burst error probability, directional antennas, analog combining network

\section{INTRODUCTION}

Vehicular traffic safety and traffic efficiency applications demand robust (reliable) communication between vehicles. Many of these applications rely on that vehicles transmit periodic status messages containing current position, speed, heading, etc. These packets are referred to as cooperative awareness messagess (CAMs) in Europe and basic safety messages (BSMs) in the US [1], [2]. The time between packets, $T$, is typically in the order of $100 \mathrm{~ms}$, but can vary due to vehicle dynamics and application requirements. Occasional packet losses are normally not problematic, since

Keerthi Kumar Nagalapur, Erik G. Ström, and Fredrik Brännström are with the Communication Systems Group, Dept. of Electrical Engineering, Chalmers University of Technology, SE-412 96, Gothenburg, Sweden, E-mail: \{keerthi, erik.strom, fredrik.brannstrom $\} @$ chalmers.se. Jan Carlsson is with Provinn $\mathrm{AB}$ and Dept. of Electrical Engineering, Chalmers University of Technology, Gothenburg, Sweden, E-mail: jan.carlsson@provinn.se. Kristian Karlsson is with the Dept. of Safety and Transport at RISE Research Institutes of Sweden, Borås, Sweden, E-mail: kristian.karlsson@ri.se.

This research has been carried out in ChaseOn in a project financed by Swedish Governmental Agency of Innovation Systems (Vinnova), Chalmers, Bluetest, Ericsson, Keysight, RISE, Smarteq, and Volvo Cars.

A part of this paper has been previously published in K. K. Nagalapur, E. G. Ström, F. Brännström, J. Carlsson, and K. Karlsson, "A simple method for robust vehicular communication with multiple nonideal antennas," in Proc. IEEE International Conference on Microwaves for Intelligent Mobility Munich, Apr. 2018. the CAMs contain information of physical quantities that vary slowly over the time duration of a few packets. However, if a number of consecutive packets from a vehicle are lost, this might lead to an application failure. Using a similar argument, the probability distribution of $K$ consecutive packet drops is used as a metric to define application-reliability in [3]. In [4], awareness-probability, defined as the probability of successfully decoding of at least $K$ packets in a tolerance timewindow, is considered to study the performance of vehicular networks. It is therefore reasonable to design the communication system to minimize the burst error probability (BrEP), i.e., the probability of incorrectly decoding $K>1$ consecutive packets, where $K$ depends on the application and $T$. This is in contrast to the more common design goal to minimize the packet error probability (PEP).

A shark fin antenna module located on top of a vehicle's roof is the standard method for housing the antennas used for vehicular communications today. However, conformal/hidden antennas are also being considered for the reasons of safety of the antennas, exterior appearance of the vehicle, and aerodynamics. Far-field functions of hidden antennas are typically directional, i.e., far from omnidirectional, due to the vehicle components that closely surround them. In fact, the antenna far-field functions might have very low, or even zero, gain in certain directions and packets arriving from an unfavorable angle-of-arrival (AOA) might be lost due to low signal-tonoise ratio (SNR). Moreover, since the vehicle position varies slowly over the time duration of a few consecutive packets, we can expect the AOA of the signal from a certain vehicle to remain approximately the same over this duration, and there is a risk of losing a number of consecutive packets from the same vehicle.

The problems due to directional antenna far-field functions can be remedied by using multiple antennas with complementing far-field functions. Combining the outputs of the multiple antennas is a well studied topic and methods such as selection combining (SC), equal gain combining (EGC), and maximal ratio combining (MRC) have been investigated thoroughly [5, Ch. 9]. The performance improvements in terms of bit error rate (BER) and/or frame error rate (FER) obtained by using these methods in vehicular scenarios has been shown in [6]-[8]. These methods either require the knowledge of the instantaneous channel amplitude and phase, or the SNR of the output signal on each antenna. Schemes that do not require the aforementioned information for combining have also been studied. A scheme called random beamforming has been explored in [9], [10], where the antenna far-field function is randomized over several time-frequency blocks to 
achieve omnidirectional coverage on average at the transmitter. A similar approach can be used at the receiver to combine the output of the antennas.

Typically, the combining methods described above require a multiport receiver (RX) to combine the signals digitally. An alternative to this approach is to use an analog combining network (ACN) consisting of analog phase shifters, variable gain amplifiers, and combiners to obtain a single combined signal that requires only a single-port RX [11], [12]. In [13], a multi antenna system that uses a hybrid precoder (a combination of digital processing and phase shifters) at the transmitter and an $\mathrm{ACN}$ at the receiver is described. A hybrid beamforming structure that uses a combination of digital processing and $\mathrm{ACN}$ at both the transmitter and receiver is studied for large-scale MIMO systems in [14]. In the above studies, the weights and the phases applied to the output of the receive antennas are computed at the RX. When the antennas and the RX are co-located, it is convenient to use a closed loop system where the information from the $\mathrm{RX}$ is used to control the analog combining network. However, from a modularity and implementation complexity perspective, it would be beneficial to devise an ACN that does not require RX feedback or knowledge of the SNR or other channel state information (CSI). This is indeed the approach taken in this paper. Moreover, since the application is traffic safety and losing a burst of consecutive packets can result in application outage leading to disastrous consequences (loss of life), we concentrate on minimizing the BrEP rather than average or peak performance.

The main contributions of this paper are:

- formulation of a general framework for the design of an ACN, which combines the outputs of $L$ antennas to a signal that is fed to a single-port receiver, that is optimized to minimize the BrEP for the worst-case AOA;

- derivation of closed-form optimal ACN parameters for $L \in\{2,3\}$ when $L \leq K$ and the PEP decreases exponentially in SNR;

- derivation of closed-form ACN parameters for $L>3$ and $L \leq K$ that guarantee that the sum of the SNRs of $K$ consecutive packets is lower bounded by the SNR for an isotropic antenna scaled with $K$ times the average of the $L$ antenna gains (which in turn guarantee that the BrEP is upper bounded by a value that is a function of the sum of the SNRs when the PEP decreases exponentially in SNR).

\section{SySTEM MODEL}

\section{A. Data Traffic Model and Time Scales}

We consider periodic transmission of CAM-like packets. That is, packets with duration $T_{\mathrm{P}}$ are transmitted every $T$ seconds. We assume that $T_{\mathrm{P}} \ll T$, which is consistent with the IEEE $802.11 \mathrm{p}$ standard based V2X communications. Indeed, for CAMs broadcasted over IEEE $802.11 \mathrm{p}$, the duration of a packet $T_{\mathrm{P}}$ is approximately $0.5-2 \mathrm{~ms}$ (the CAM packet sizes of varies between approximately 400 to 1500 byte and the data rate is fixed to $6 \mathrm{Mbit} / \mathrm{s}[15])$. Hence, $T_{\mathrm{P}}$ is very small in comparison to $T$, which varies between $100-1000 \mathrm{~ms}$ [1, Table

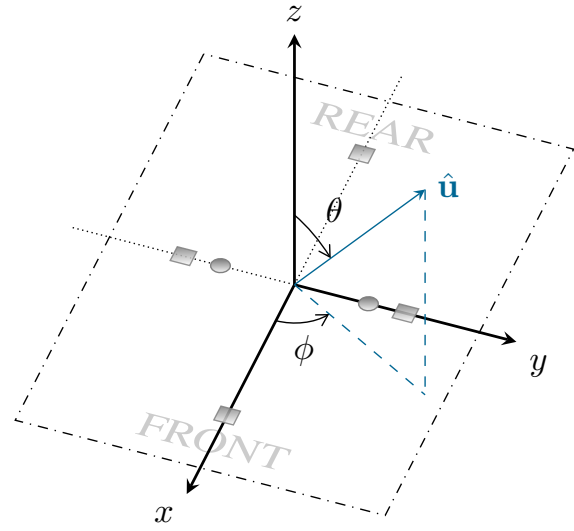

Fig. 1. Antenna coordinate system. The roof of the vehicle is in the $x y$ plane.

1]. We declare an application outage when $K$ consecutive packets are lost, i.e., when the receiver has not decoded any packets for $K T$ seconds. Therefore, we design a time-varying ACN to minimize the probability of application outage. The design is optimal under the condition that the change in AOA is negligible over $K T$ seconds and the time-varying changes in the ACN are negligible over $T_{\mathrm{P}}$. The exact statement of these assumptions is made more precise below.

\section{B. Antennas}

Consider $L \geq 2$ antennas located on a vehicle. All the antennas are assumed to be at the same height from the ground and in the $x y$ plane as shown in Fig. 1. The angles $\phi$ and $\theta$ are the azimuth and polar angles, respectively, and the vehicle orientation with respect to the coordinate system is also shown in the figure. Two examples of antenna placement are also shown in the figure (circles and squares).

Let $g_{l}(\phi)$ be the far-field function of Antenna $l \in$ $\{0,1, \ldots, L-1\}$ in the azimuth plane. The far-field function is normalized such that $\left|g_{l}(\phi)\right|^{2}$ represents the relative directive gain of Antenna $l$ with respect to an isotropic antenna. We assume that the antennas are vertically polarized and that the incident electrical field is composed of a number of vertically polarized plane waves (multipath components) arriving in the azimuth plane, i.e., with the polar angle $\theta=\pi / 2$.

\section{Propagation Environment and Antenna Output Signals}

The performance of the antenna system depends on the propagation environment. Since the focus of the paper is to provide robustness, i.e., to improve the worst-case performance, we study the system under the worst-case propagation conditions. It is intuitively clear that the worst-case propagation condition from an antenna system perspective is when the incident field consist of multipath components arriving from the worst-case AOA. That most of the power comes from a single AOA is a reasonable model for highway environments, which typically have a dominating line-of-sight component or a few strong scatterers [16], [17]. In Appendix A, we briefly discuss the performance of the proposed combining scheme when there are many multipath components arriving to the receiver. 
For simplicity, we assume that a single multipath component $^{1}$ is impinging on the antenna configuration from AOA $\phi(t)$. We assume that the geometry and mobility of the transmitter, receiver, and potential scatterers is such that the change in AOA is negligible over $K T$ seconds, and therefore omit the explicit time dependence from the notation. The complex-valued channel gain at Antenna $l$ is then given by [18, (8)]

$$
h_{l}(t)=\tilde{a}(t) g_{l}(\phi) e^{-j \tilde{\Omega}_{l}(t)}
$$

where $\tilde{a}(t)$ is the complex-valued gain and $\tilde{\Omega}_{l}(t)$ is the distance-induced phase shift at Antenna $l$, i.e., $\tilde{\Omega}_{n, l}(t)=$ $(2 \pi / \lambda) d_{l}(t)$, where $d_{l}(t)$ is the time-varying propagation distance from the transmitter to Antenna $l$ and $\lambda$ is the wavelength of the carrier signal. The model in (1) is obtained from $[18,(8)]$ by setting the number of multipath components to one. Since we are interested in optimizing the ACN at the receiver, the angle-of-departure (AOD) of the single multipath component at the transmitter is irrelevant and the transmitter antenna gain at AOD of the single component is captured by $\tilde{a}(t)$. Consequently, considering the Antenna 0 as the reference, the channel gain at Antenna $l$ can be written as

$$
h_{l}(t)=a(t) g_{l}(\phi) e^{-\jmath \Omega_{l}(t)},
$$

where $a(t)=\tilde{a}(t) e^{-\jmath \tilde{\Omega}_{0}(t)}$ and $\Omega_{l}(t)=\tilde{\Omega}_{l}(t)-\tilde{\Omega}_{0}(t)$ is the phase difference at Antenna $l$ with respect to the reference antenna. Under the above assumption of slowly varying AOA, $\Omega_{l}(t)$ is approximately constant over $K T$ seconds. Dropping the time-dependency of $\Omega_{l}(t)$, we arrive at

$$
h_{l}(t)=a(t) g_{l}(\phi) e^{-\jmath \Omega_{l}},
$$

and the signal at the output of the $l$ th antenna under the narrowband assumption is given by

$$
r_{l}(t)=s(t) h_{l}(t)+n_{l}(t),
$$

where $s(t)$ is the transmitted signal and $n_{l}(t)$ for $l=$ $0,1, \ldots, L-1$ are independent identically distributed complex additive white Gaussian noise (AWGN) processes with power $\mathbb{E}\left\{\left|n_{l}(t)\right|^{2}\right\}=P_{\mathrm{n}}$ over the bandwidth of the signal $s(t)$.

\section{Analog Combining Network}

We restrict the ACN to consist of analog phase shifters and an adder as seen in Fig. 2. The output of the combiner $r(t)$ is given by

$$
r(t)=\sum_{l=0}^{L-1} r_{l}(t) e^{\jmath \varphi_{l}(t)},
$$

where $\varphi_{l}(t)$ is the time-varying phase shift applied to the $l$ th antenna output and $\varphi_{0}(t)=0$. For simplicity, we let

$$
\varphi_{l}(t)=\alpha_{l} t+\beta_{l}, \quad l=0,1, \ldots, L-1 .
$$

Hence, $\alpha_{l}$ is the phase slope and $\beta_{l}$ is the phase offset of the $l$ th phase shifter, and $\alpha_{0}=\beta_{0}=0$ (since $\varphi_{0}(t)=0$ ). By

\footnotetext{
${ }^{1}$ The extension to multiple components from the same AOA is straight forward, but complicates notation and does not change the development of the optimum ACN.
}

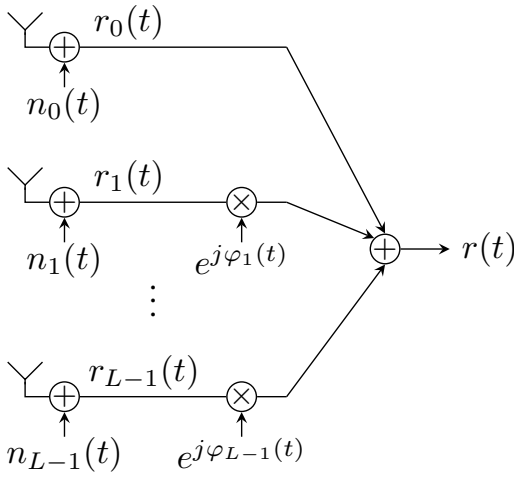

Fig. 2. The analog combining network with $L$ antennas.

combining (4) and (5), we can write the output of the ACN as

$$
\begin{aligned}
r(t) & =s(t) a(t) \sum_{l=0}^{L-1} g_{l}(\phi) e^{-\jmath\left(\Omega_{l}-\alpha_{l} t-\beta_{l}\right)}+\sum_{l=0}^{L-1} \tilde{n}_{l}(t) \\
& =s(t) a(t) g(\phi, \boldsymbol{\alpha}, \boldsymbol{\beta}, t)+\sum_{l=0}^{L-1} \tilde{n}_{l}(t),
\end{aligned}
$$

where

$$
g(\phi, \boldsymbol{\alpha}, \boldsymbol{\beta}, t)=\sum_{l=0}^{L-1} g_{l}(\phi) e^{-\jmath\left(\Omega_{l}-\alpha_{l} t-\beta_{l}\right)}
$$

is the effective time-varying antenna far-field function, $\alpha=$ $\left[\alpha_{1}, \ldots, \alpha_{L-1}\right]^{\top}, \boldsymbol{\beta}=\left[\beta_{1}, \ldots, \beta_{L-1}\right]^{\top}$, and $\tilde{n}_{l}(t)=n_{l}(t) e^{\jmath \phi_{l}}$ has the same distribution as $n_{l}(t)$ since $n_{l}(t)$ is circularly symmetric.

To avoid complicating channel estimation, we choose $\alpha_{l}$ for all $l$ to be small enough such that the phase change over a packet duration $T_{\mathrm{P}}$ is negligible, which implies that the effective far-field function during the $k$ th packet can be approximated to be $g(\phi, \boldsymbol{\alpha}, \boldsymbol{\beta}, k T)$. Consequently, the average $\mathrm{SNR}$ of the $k$ th packet is given by

$$
\begin{aligned}
\bar{\gamma}(\phi, \boldsymbol{\alpha}, \boldsymbol{\beta}, k) & =\frac{\mathbb{E}\left\{|a(t) s(t)|^{2}\right\}|g(\phi, \boldsymbol{\alpha}, \boldsymbol{\beta}, k T)|^{2}}{\mathbb{E}\left\{\left|\sum_{l=0}^{L-1} \tilde{n}_{l}(t)\right|^{2}\right\}} \\
& =\frac{P_{\mathrm{r}}}{L P_{\mathrm{n}}}|g(\phi, \boldsymbol{\alpha}, \boldsymbol{\beta}, k T)|^{2},
\end{aligned}
$$

where we have assumed that the change in pathloss and largescale fading is negligible for $K T$ seconds, implying that the average received signal power $\mathbb{E}\left\{|a(t) s(t)|^{2}\right\}=P_{\mathrm{r}}$ is the same for $K$ consecutive packets.

\section{Burst ERror ProbABILITY}

In this section, we formulate the problem of designing the ACN to minimize the BrEP. The PEP of the $k$ th packet is a function of the average SNR and is denoted by $P_{\mathrm{e}}(\bar{\gamma}(\phi, \boldsymbol{\alpha}, \boldsymbol{\beta}, k))$. The function $P_{\mathrm{e}}(\cdot)$ depends on the modulation and coding scheme used, the length of the packet, and the characteristics of the channel. As mentioned earlier, we intend to minimize the probability of having a burst of $K$ consecutive packet errors denoted by $P_{\mathrm{B}}(\phi, \boldsymbol{\alpha}, \boldsymbol{\beta}, K)$. Assuming that the 
packet errors are independent, the BrEP is the product of the individual PEPs of the $K$ consecutive packets and is given by

$$
P_{\mathrm{B}}(\phi, \boldsymbol{\alpha}, \boldsymbol{\beta}, K)=\prod_{k=0}^{K-1} P_{\mathrm{e}}(\bar{\gamma}(\phi, \boldsymbol{\alpha}, \boldsymbol{\beta}, k)) .
$$

Since we are interested in determining the optimum $\alpha$ that minimizes the BrEP for the worst-case AOA $\phi \in[0,2 \pi)$, we formulate the following optimization problem.

$$
\boldsymbol{\alpha}^{*}=\underset{\left\{\alpha_{l}\right\} \in \mathbb{R}}{\arg \inf } \sup _{\phi, \beta_{l} \in[0,2 \pi)} P_{\mathrm{B}}(\phi, \boldsymbol{\alpha}, \boldsymbol{\beta}, K) .
$$

Note that we maximize the BrEP with respect to $\boldsymbol{\beta}$ in addition to $\phi$ to include the effect of the worst-case initial offset of $\varphi_{l}(t)$. The optimization problem considers the worst-case AOA and $\boldsymbol{\beta}$ for each $\boldsymbol{\alpha}$ while finding $\boldsymbol{\alpha} *$.

We now have a framework to find $\boldsymbol{\alpha}^{*}$ that minimizes the BrEP for arbitrary far-field functions of the antennas and PEP functions when the signal arrives at the RX as a single component or when the spread of the AOA is very small. It might not be possible to solve the optimization problem in (9) analytically for any given far-field function and PEP function, in which case numerical optimization can be used.

As a special case of PEP function, we consider an exponential PEP function of the form $P_{\mathrm{e}}(\bar{\gamma})=a \exp (-b \bar{\gamma})$, where $a, b>0$ are constants. The BrEP in the case of the exponential PEP function is given by

$$
P_{\mathrm{B}}(\phi, \boldsymbol{\alpha}, \boldsymbol{\beta}, K)=\prod_{k=0}^{K-1} a e^{-b \bar{\gamma}(\phi, \boldsymbol{\alpha}, \boldsymbol{\beta}, k)} .
$$

The optimization problem in (9) can then be written as

$$
\begin{aligned}
& \boldsymbol{\alpha}^{*}=\underset{\left\{\alpha_{l}\right\} \in \mathbb{R}}{\arg \inf } \sup _{\phi,\left\{\beta_{l}\right\} \in[0,2 \pi)} \ln \left(P_{\mathrm{B}}(\phi, \boldsymbol{\alpha}, \boldsymbol{\beta}, K)\right)
\end{aligned}
$$

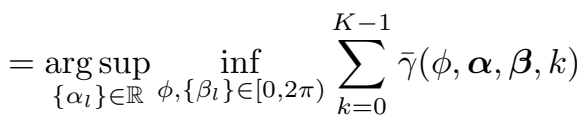

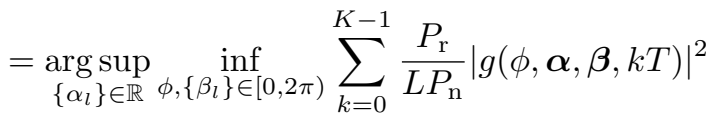

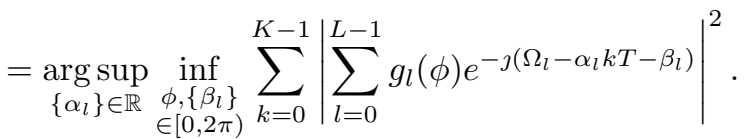

If we let $\psi_{l}=\bmod \left(\Omega_{l}-\beta_{l}-\angle g_{l}(\phi), 2 \pi\right)$, where $\bmod (u, v)$ is the remainder after dividing $u$ by $v$, then $\beta_{l} \in[0,2 \pi)$ implies that $\psi_{l} \in[0,2 \pi)$ and the optimization problem can be written as

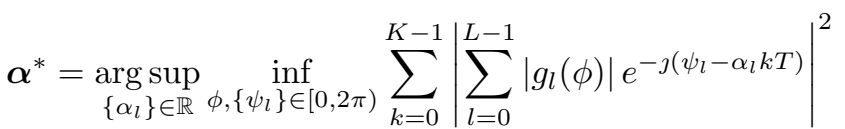

$$
\begin{aligned}
& =\underset{\left\{\alpha_{l}\right\} \in \mathbb{R}}{\arg \sup } \inf _{\phi,\left\{\psi_{l}\right\} \in[0,2 \pi)} J(\phi, \boldsymbol{\alpha}, \boldsymbol{\psi}, K),
\end{aligned}
$$

where the objective function $J(\phi, \boldsymbol{\alpha}, \boldsymbol{\psi}, K)$ is found through algebraic manipulation of (13) to be

$$
\begin{aligned}
& J(\phi, \boldsymbol{\alpha}, \boldsymbol{\psi}, K)=K \sum_{l=0}^{L-1}\left|g_{l}(\phi)\right|^{2}+2 \sum_{l=0}^{L-2} \sum_{m=l+1}^{L-1} \\
& \left|g_{l}(\phi)\right|\left|g_{m}(\phi)\right| \sum_{k=0}^{K-1} \cos \left(\psi_{m}-\psi_{l}-\left(\alpha_{m}-\alpha_{l}\right) k T\right) .
\end{aligned}
$$

Theorem 1. The optimum of the objective function for an arbitrary $\phi$

$$
J^{*}(\phi) \triangleq \sup _{\boldsymbol{\alpha}} \inf _{\boldsymbol{\psi}} J(\phi, \boldsymbol{\alpha}, \boldsymbol{\psi}, K),
$$

is lower bounded as

$$
J^{*}(\phi) \geq K \sum_{l=0}^{L-1}\left|g_{l}(\phi)\right|^{2} \quad \text { when } L \leq K,
$$

and the solutions

$$
\begin{gathered}
\alpha_{0}^{*}=0,\left(\left(\alpha_{m}^{*}-\alpha_{l}^{*}\right) T / 2\right) \in \mathcal{X}^{*} \text { for } 0 \leq l<m \leq L-1, \\
\mathcal{X}^{*} \triangleq\{q \pi / K: q \in \mathbb{Z}\} \backslash\{q \pi: q \in \mathbb{Z}\},
\end{gathered}
$$

guarantee the lower bound. The solution in (18) with the smallest nonnegative phase slopes is

$$
\alpha_{l}^{*}=\frac{l 2 \pi}{K T}, \quad l=1,2, \ldots, L-1 .
$$

Furthermore, for $L=2$ and 3, the bound in (17) is tight and the solutions in (18) are optimal.

Proof: See Appendix B.

In summary, Theorem 1 shows that by using the solutions in (18) and (19) it is possible to guarantee a minimum value for the objective function and hence limit the BrEP to a maximum value for all AOAs including the worst-case AOA.

Example 1. For $L=5$ and $K=5$, a solution set that achieves the lower bound in (17) is given by $\left[\alpha_{1}^{*}, \alpha_{2}^{*}, \alpha_{3}^{*}, \alpha_{4}^{*}\right]=$ $[2 \pi /(K T), 4 \pi /(K T), 6 \pi /(K T), 8 \pi /(K T)]$ and $\alpha_{0}^{*}=0$ as the output of the $l=0$ antenna is not phase shifted.

In the case of $L>3$, proving the tightness of the bound in (17) seems to be analytically intractable. In such a case, the optimization problem (14) can be solved numerically when $L$ is not large.

When the phase slopes $\alpha_{l}^{*}$ in (18) are used, the objective is independent of $\psi_{l}$ and hence independent of $\beta_{l}$. Therefore, for any initial offset $\beta_{l}$ the worst-case $\mathrm{AOA} \phi$ that results in the highest BrEP is given by

$$
\phi^{\star}=\underset{\phi \in[0,2 \pi)}{\arg \min } \sum_{l=0}^{L-1}\left|g_{l}(\phi)\right|^{2} .
$$

As a consequence, when the proposed combining scheme is used to minimize the BrEP in case of multiple directional antennas, the antennas should be designed and oriented such that $\sum_{l=0}^{L-1}\left|g_{l}\left(\phi^{\star}\right)\right|^{2}$ is maximized.

\section{A. Two Antenna Case}

In this section, a few aspects specific to the $L=2$ antenna case are discussed. 
1) Different repetition periods: the optimum phase slope $\alpha^{*}=\alpha_{1}^{*}$ for $L=2$ antennas and a given $K$ and $T$ is not unique, see (18). This allows a choice of $\alpha^{*}$ that is the optimum for several different repetition periods. Indeed, (18) tells us that $\alpha^{*}=2 \pi /\left(K T_{1}\right)$ is optimum for the period $T=T_{1}$. However, since

$$
\alpha^{*}=\frac{2 \pi}{K\left(r T_{1}\right)} r
$$

it follows from (18) that $\alpha^{*}$ is also optimum for period $T=$ $r T_{1}$, where $r \in \mathbb{N} \backslash\{K, 2 K, \ldots\}$.

Example 2. Let $T_{1}=0.1 \mathrm{~s}$ and $T_{2}=0.3 \mathrm{~s}$ be the periods of the messages arriving at the RX from two different transmitters. Suppose $K=5$, the optimum rate of phase shift $\alpha^{*}=2 \pi /\left(K T_{1}\right)$ is optimum for both periods.

2) Similarity to EGC: in EGC, signals from the two antennas are phase aligned or co-phased before they are added together to increase the SNR. This co-phasing can be achieved by phase shifting the output of the $l=1$ antenna $r_{1}(t)$ and adding it to the output of the reference antenna $r_{0}(t)$. In the proposed combining scheme, $r_{1}(t)$ is phase shifted continuously and added to $r_{0}(t)$. When $\alpha^{*}=2 \pi /(K T)$, the signal corresponding to the $K$ consecutive packets at the $l=1$ antenna is shifted with $K$ different phases that uniformly sample the domain $[0,2 \pi)$. Therefore, $\alpha^{*}$ minimizes the phase difference between the signals at the two antennas during one of the $K$ consecutive packets. The deviation from perfect cophasing is dependent on the initial phase offset $\beta=\beta_{1}$. As $K$ increases, the phase difference during one of the $K$ consecutive packets decreases and the output average SNR of one of the $K$ packets reaches close to the case of EGC.

\section{COMPARISON WITH STANDARD SCHEMES}

In this section, the performance of the proposed combining scheme is compared with a few standard combining schemes. In the case of the exponential PEP function considered in Section III, minimizing the BrEP is equivalent to maximizing the sum of the average SNRs of the $K$ packets as seen in (11), hereafter referred as the sum-SNR. Therefore, the sum-SNR is used as a performance criterion to compare the performance of the combining schemes.

1) Single antenna: the sum-SNR at the output of Antenna $l$ is given by

$$
\rho_{l}(\phi)=\sum_{k=0}^{K-1} \bar{\gamma}_{l}(\phi, k)=\frac{K P_{\mathrm{r}}}{P_{\mathrm{n}}}\left|g_{l}(\phi)\right|^{2} .
$$

When the antenna is omnidirectional in the azimuth plane, the sum-SNR is given by

$$
\rho_{l, \mathrm{OMN}}(\phi)=\frac{K P_{\mathrm{r}} G_{l}}{P_{\mathrm{n}}},
$$

where $G_{l}$ is the directive gain of Antenna $l$ in all the directions. In the case of an isotropic antenna with $G_{l}=$ $1, \rho_{l, \mathrm{ISO}}(\phi)=K P_{\mathrm{r}} / P_{\mathrm{n}}$.

2) MRC: this scheme requires $L$ RF-chains and analog to digital converters (ADCs), and a multiple port receiver that estimates the complex-valued channel gains and performs combining digitally. The sum-SNR is given by

$$
\rho_{\mathrm{MRC}}(\phi)=\sum_{k=0}^{K-1} \bar{\gamma}_{\mathrm{MRC}}(\phi, k)=\frac{K P_{\mathrm{r}}}{P_{\mathrm{n}}}\left(\sum_{l=0}^{L-1}\left|g_{l}(\phi)\right|^{2}\right) \text {. }
$$

3) EGC: this scheme requires $L$ RF-chains and ADCs, and a multiple port receiver that estimates the channel phases and performs combining digitally. The sum-SNR is given by

$$
\rho_{\mathrm{EGC}}(\phi)=\sum_{k=0}^{K-1} \bar{\gamma}_{\mathrm{EGC}}(\phi, k)=\frac{K P_{\mathrm{r}}}{L P_{\mathrm{n}}}\left(\sum_{l=0}^{L-1}\left|g_{l}(\phi)\right|\right)^{2} .
$$

4) SC: this scheme requires $L \mathrm{RF}$-chains, and a digital or analog circuitry to measure the SNRs on each branch and choose a branch. The sum-SNR is given by

$$
\rho_{\mathrm{SC}}(\phi)=\sum_{k=0}^{K-1} \bar{\gamma}_{\mathrm{SC}}(\phi, k)=\frac{K P_{\mathrm{r}}}{P_{\mathrm{n}}} \max _{l}\left\{\left|g_{l}(\phi)\right|^{2}\right\} .
$$

5) $\mathrm{ACN}$ : the proposed scheme requires analog phase shifters on $L-1$ branches operating independently and a combiner. The sum-SNR when the solution in (18) is used is given by

$$
\begin{aligned}
\rho_{\mathrm{ACN}}(\phi) & =\sum_{k=0}^{K-1} \bar{\gamma}\left(\phi, \boldsymbol{\alpha}^{*}, \boldsymbol{\beta}, k\right) \\
& =\frac{K P_{\mathrm{r}}}{L P_{\mathrm{n}}}\left(\sum_{l=0}^{L-1}\left|g_{l}(\phi)\right|^{2}\right), \forall \beta_{l} \in[0,2 \pi) .
\end{aligned}
$$

The sum-SNR in the case of MRC and ACN are relate as $\rho_{\mathrm{MRC}}(\phi)=L \rho_{\mathrm{ACN}}(\phi)$.

The MRC scheme outperforms EGC, SC, and ACN for any far-field functions $g_{l}(\phi)$. The relative performance of SC and EGC for an AOA $\phi$ depends on the far-field functions $g_{l}(\phi)$. The sum-SNR of MRC, EGC, and SC schemes is higher compared to our ACN scheme, implying lower BrEP. However, these schemes require additional hardware and/or signal processing as mentioned above.

\section{Numerical RESUlts}

In this section, the performance of the ACN is studied by using example antenna far-field functions. The sum-SNR $\rho(\phi)$ discussed in Section IV is used to illustrate the direction dependency of the BrEP. The $\rho_{l}(\phi)$ of Antenna $l$ is directly proportional to $\left|g_{l}(\phi)\right|^{2}$ and therefore it also serves the purpose of visualizing the AOA dependent gain of the antenna.

\section{A. Two Monopoles on a Vehicle Roof}

We consider the measured far-field functions of two monopole antennas placed on the roof of a Volvo XC90 to analyze the performance of the ACN. The $l=0$ and $l=1$ monopole antennas are located at $(x, y)=(0,0.4 \mathrm{~m})$ and $(0,-0.4 \mathrm{~m})$, respectively (indicated by circles in Fig. 1$)$. The $\rho_{0}(\phi)$ and $\rho_{1}(\phi)$ of the two monopole antennas are shown in Fig. 3. The $\rho(\phi)$ have been plotted by setting $P_{\mathrm{r}} / P_{\mathrm{n}}=1$ and $K=5$. As seen in the figure, both the antennas exhibit very 


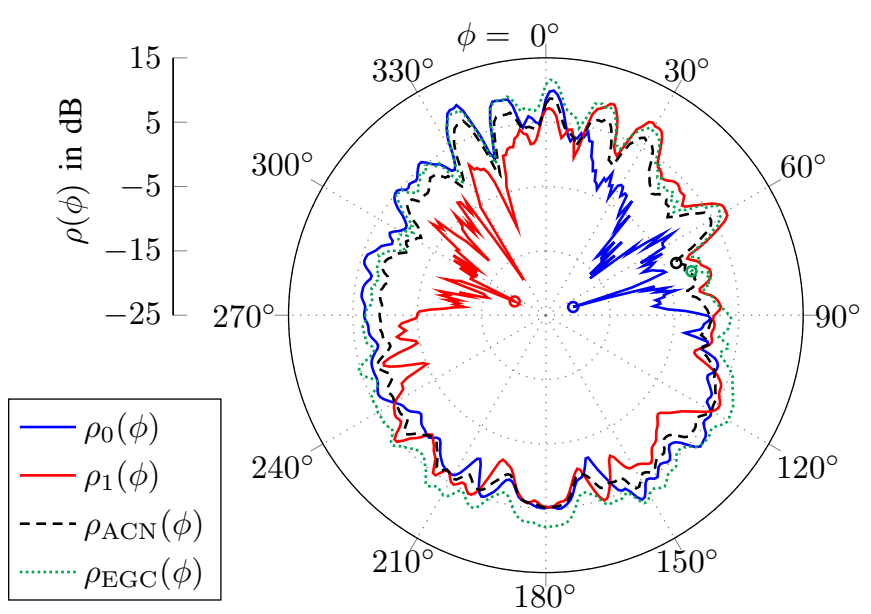

Fig. 3. Sum-SNR $\rho(\phi)$ of the two individual roof-top monopoles and sumSNR after combining with ACN and EGC. Here, $P_{\mathrm{r}} / P_{\mathrm{n}}=1$ and $K=5$. The worst-case sum-SNR is marked with circles.

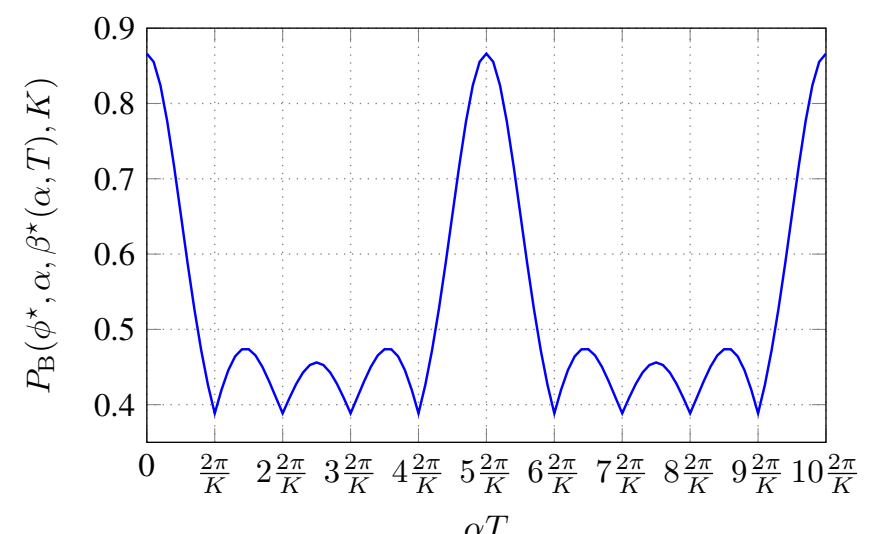

Fig. 4. BrEP as a function of $\alpha T$ for a fixed $\mathrm{AOA} \phi^{\star} \approx 68^{\circ}$ and worst-case $\beta$ using the two roof-top monopole antennas, $P_{\mathrm{r}} / P_{\mathrm{n}}=10$, and $K=5$.

low $\rho(\phi)$ at certain AOAs. If only one of the two antennas is used, the packets arriving in the AOAs of low $\rho(\phi)$ will, of course, have high BrEP. The sum-SNR can be improved by combining the output of the antennas using the proposed ACN. As seen in the figure, $\rho_{\mathrm{ACN}}(\phi)$ is (as expected) sandwiched between the two individual antenna sum-SNRs, $\rho_{0}(\phi)$ and $\rho_{1}(\phi)$. Hence, the ACN gives improved robustness against unfavorable AOA. The sum-SNRs for EGC is also shown in the figure, while the sum-SNRs for MRC and SC have been omitted. However, the latter are related to the plots in the figure through the relation $\rho_{\mathrm{MRC}}(\phi)=2 \rho_{\mathrm{ACN}}(\phi)$ and $\rho_{\mathrm{SC}}(\phi)=\max \left\{\rho_{0}(\phi), \rho_{1}(\phi)\right\}$.

Comparing the sum-SNRs of the antenna arrangements at worst-case AOAs in Fig. 3, we note that the ACN is $17.4 \mathrm{~dB}$ and $16.5 \mathrm{~dB}$ better than Antenna 0 and Antenna 1, respectively. The ACN is $1.81 \mathrm{~dB}$ worse than EGC, $1.83 \mathrm{~dB}$ worse than $\mathrm{SC}$, and $3 \mathrm{~dB}$ worse than MRC. Hence, the ACN provides a tremendous gain in robustness compared to choosing just one of the antennas. The performance loss against the standard combining schemes is relatively modest, especially considering that a 2-port receiver is needed for these schemes.

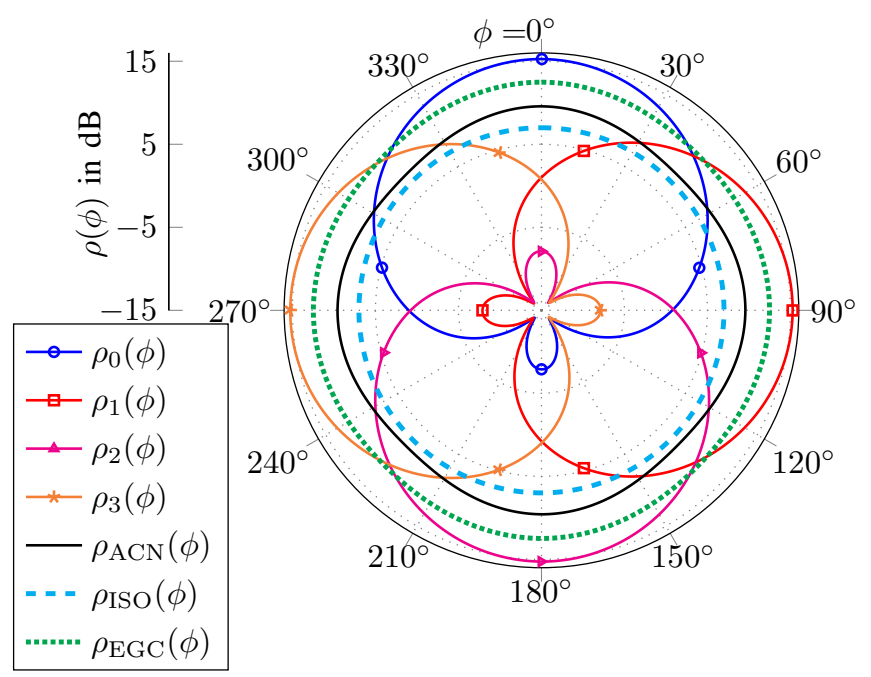

Fig. 5. $\rho(\phi)$ of the four patch antennas. The patch antennas exhibit directional power gains. $P_{\mathrm{r}} / P_{\mathrm{n}}=1$ and $K=5$.

$\multimap$ Ant. $0 \multimap$ Ant. $1 \multimap$ Ant. $2 \multimap$ Ant. 3

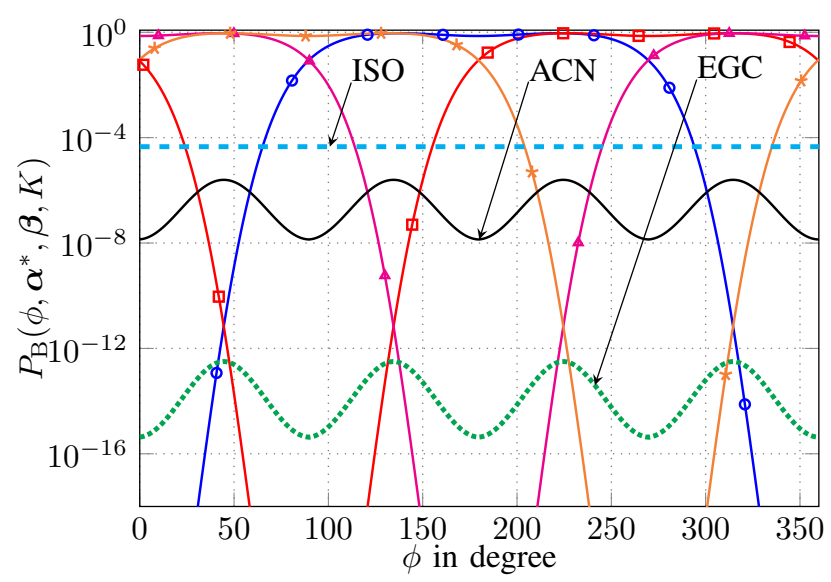

Fig. 6. BrEP as a function of AOA $\phi$ for the individual antennas and the combined output when $K=5,\left[\alpha_{1}^{*}, \alpha_{2}^{*}, \alpha_{3}^{*}\right]=$ $[2 \pi /(K T), 4 \pi /(K T), 6 \pi /(K T)]$.

The performance of the ACN for an suboptimum choice of $\alpha$ is shown in Fig. 4. The previous setup of the two monopole antennas with $K=5$ and $P_{\mathrm{r}} / P_{\mathrm{n}}=10 \mathrm{~dB}$ is used. We consider the exponential PEP function $P_{\mathrm{e}}(\bar{\gamma})=\exp (-\bar{\gamma} / 5)$. For $L=2$, the sum-SNR and, therefore, the BrEP is a function of $\alpha T$ and $\beta$, see (12). Fig. 4 plots the BrEP versus $\alpha T$ for the worst-case AOA $\phi^{\star} \approx 68^{\circ}$ (marked by a circle in Fig. 3 ). The worst-case initial offset $\beta^{\star}(\alpha T)$ is used for every $\alpha T$. We note that the BrEP is minimized when $\alpha T=u(2 \pi / K)$ where $u \in\{1,2, \ldots\} \backslash\{K, 2 K, \ldots\}$, which is in agreement with the solution in (18). An $\alpha^{\prime}=2 \pi /\left(K T^{\prime}\right)$ designed for $T=T^{\prime}$ is optimum for several integer multiples of $T^{\prime}$ and this result agrees with the discussion in Section III-A1. It can also be observed that the deviation of the BrEP from the minima is not significant for a large range of $\alpha T$. Therefore, the ACN can handle small mismatches in $\alpha$ or $T$ without significant performance loss. 


\section{B. Four Patch Antennas on a Vehicle Roof}

As an example of $L>2$, we consider $L=4$ patch antennas. The antennas $l=0,1,2$, and 3 are located at $(x, y)=$ $(1 \mathrm{~m}, 0 \mathrm{~m}),(0 \mathrm{~m}, 0.6 \mathrm{~m}),(-1 \mathrm{~m}, 0 \mathrm{~m})$, and $(0 \mathrm{~m},-0.6 \mathrm{~m})$, respectively (indicated by squares in Fig. 1). The antennas are oriented such that the E-plane far-field function of each antenna coincides with the $x y$ plane and the perpendiculars to the ground planes pass through the origin of the coordinate system. The width, length, and height of the patch antennas are $0.5 \lambda / \sqrt{\epsilon_{\mathrm{r}}}, 0.5 \lambda / \sqrt{\epsilon_{\mathrm{r}}}$, and $0.05 \lambda / \sqrt{\epsilon_{\mathrm{r}}}$, respectively, where $\lambda$ is the wavelength of the carrier with frequency $5.9 \mathrm{GHz}$ and $\epsilon_{\mathrm{r}}=2.2$ is the dielectric constant of the substrate. The length and width of the ground plane is equal to $\lambda$. The far-field functions of the patch antennas are obtained using the method of moments. The sum-SNR $\rho(\phi)$ of the antennas is shown in Fig. 5, $P_{\mathrm{r}} / P_{\mathrm{n}}=1$ and $K=5$ are used. It can be observed that a single patch antenna exhibits very low $\rho(\phi)$ for a large range of AOAs in the azimuth plane, implying higher BrEP at these AOAs. The ACN can be used to combine the outputs of the four antennas to minimize the BrEP in these AOAs. The phase slopes for the three antennas are chosen according to (19), i.e., $\alpha_{l}^{*}=l 2 \pi /(K T)$, for $l=1,2,3$. It can be observed that $\rho_{\mathrm{ACN}}(\phi)$ of the ACN has higher values for AOAs where the individual antennas have very low values. The sum-SNR in the case of a single isotropic antenna and in the case of the patch antennas combined using EGC are also shown in the figure. The plots corresponding to MRC and SC have been omitted in the figure. However, they are related to the plots in the figure through the relation $\rho_{\mathrm{MRC}}(\phi)=4 \rho_{\mathrm{ACN}}(\phi)$ and $\rho_{\mathrm{SC}}(\phi)=\max \left\{\rho_{0}(\phi), \rho_{1}(\phi), \rho_{2}(\phi), \rho_{3}(\phi)\right\}$.

Comparing the sum-SNRs of the antenna arrangements at worst-case AOAs in Fig. 5, we note that the ACN is much better than Antenna 0 (or any of the other individual antennas), which is not surprising since the patch antennas are not designed to be omnidirectional (as opposed to the monopoles in Fig. 3). The $\mathrm{ACN}$ is $3.49 \mathrm{~dB}$ worse than EGC, $3.00 \mathrm{~dB}$ worse than $\mathrm{SC}, 6 \mathrm{~dB}$ worse than MRC, but $1.10 \mathrm{~dB}$ better than an isotropic antenna. In fact, the ACN is better than the isotropic antenna for all AOAs. Again, comparing with the standard combining schemes is not fair, since a 4-port receiver is needed for these schemes.

The BrEP in the setup of the four patch antennas as a function of AOA is shown in Fig. 6. An exponential PEP function $P_{\mathrm{e}}(\bar{\gamma})=\exp (-\bar{\gamma} / 5)$ is used and $P_{\mathrm{r}} / P_{\mathrm{n}}=10 \mathrm{~dB}$. As in the case of $L=2$, the BrEP of the individual antennas is close to 1 for the AOAs that have very low $\rho(\phi)$. The BrEP for the AOAs corresponding to low gains in the individual antennas is reduced by the $\mathrm{ACN}$. The $\mathrm{ACN}$ enables robust communication for signals from all AOAs. The figure also shows the BrEP in the case of a single isotropic antenna and in the case of the patch antennas combined using EGC, the $\mathrm{BrEP}$ in these cases is in agreement with their $\rho(\phi)$ in Fig. 5.

\section{Numerical Optimization}

The optimization problem in (9) may not be analytically tractable for an arbitrary PEP function, $K$, and $L$. In such a scenario, numerical optimization can be used to find the optimal phase slopes for the ACN. We considered the optimization problem in the case of the two measured monopole antennas and $K=5$ with two PEP functions for uncoded Gray-coded QPSK with independent bit errors [19, Ch. 6], namely

$$
\begin{aligned}
\text { AWGN: } P_{\mathrm{e}}(\bar{\gamma}) & =1-(1-Q(\sqrt{\bar{\gamma}}))^{N_{\mathrm{b}}}, \\
\text { Rayleigh fading: } P_{\mathrm{e}}(\bar{\gamma}) & =1-\left(\frac{1}{2}+\frac{1}{2} \sqrt{\frac{\bar{\gamma}}{2+\bar{\gamma}}}\right)^{N_{\mathrm{b}}},
\end{aligned}
$$

where $N_{\mathrm{b}}$ is the number of bits in the packet and $\bar{\gamma}$ is the average SNR. Exhaustive search was used to solve the optimization problem numerically with $N_{\mathrm{b}}=3200$ and $P_{\mathrm{r}} / P_{\mathrm{n}}=10 \mathrm{~dB}$. The analytically obtained optimum solution $\alpha^{*}=2 \pi /(K T)$ in the case of exponential PEP function was found to be the optimum solution. We conjecture that the optimal solution in (18) is optimal for other monotonically decreasing PEP functions.

\section{CONCLUSIONS}

In this paper, we have proposed a simple method consisting of phase shifters to combine the outputs of $L$ directional antennas to enable robust vehicle-to-vehicle communications with a single-port receiver. To guarantee robustness, we have designed our method to minimize the burst error probability, i.e., the probability of $K$ consecutive packet errors for the worst-case angle of arrival. The combining scheme does not need knowledge of the instantaneous complex-valued channel gains or the SNRs on each antenna branch in contrast to the standard combining schemes. We have used measured far-field functions of two monopole antennas mounted on a vehicle and other example far-field functions to show the benefits of the scheme. For example, the ACN gives a gain of more than $16.5 \mathrm{~dB}$ in worst-case sum-SNR compared to using just one of the two realized monopole antennas. For the simulated patch antennas, it is shown that the ACN gives a quite smooth and relatively large sum-SNR for all angle-of-arrivals.

The phase slopes that guarantee an upper bound on the BrEP are derived in the case of $L \leq K$ and are given by $\alpha_{l}=l 2 \pi /(K T), l=1,2, \ldots, L-1$. Furthermore, it is shown that that the upper bound is indeed tight for the case of $L=2$ and 3 , which implies that the solution is optimum for this case. Moreover, for $L=2$, the optimum phase slope designed for a specific $T$ is optimum when the actual period is certain multiples of $T$ and robust to a large range of other periods. It is interesting to note that the optimum phase slopes are independent of the antenna placement and far-field functions, which makes the method very robust to manufacturing tolerances.

The proposed scheme is also relevant for low cost sensor nodes with strict requirements on power consumption and complexity. Multiple low cost antennas with directional farfield functions can be used and combined using the proposed method to support robust communications.

\section{APPENDIX A}

\section{Rich Multipath Propagation}

In this appendix, we consider rich multipath propagation, i.e., when a large number of multipath components distributed 
uniformly in the azimuth plane are impinging on the antenna system. The channel gains $h_{l}(t)$ can then be accurately modeled as uncorrelated complex Gaussian processes with zero mean when the following conditions are satisfied: (i) the separation between the antennas is larger than $\lambda$, (ii) a dominant component is absent, and (iii) the antennas are assumed to be omnidirectional [20, Sec. 5.4]. The requirement of omnidirectional antennas in assumption (iii) can be relaxed when the antennas have a broad beamwidth or when the main lobes of the antennas are oriented in different directions.

The signal at the output of the combiner is given by

$$
\begin{aligned}
r(t) & =s(t) \sum_{l=0}^{L-1} h_{l}(t) e^{\jmath\left(\alpha_{l} t+\beta_{l}\right)}+\sum_{l=0}^{L-1} n_{l}(t) e^{\jmath\left(\alpha_{l} t+\beta_{l}\right)} \\
& =s(t) \sum_{l=0}^{L-1} \tilde{h}_{l}(t)+\sum_{l=0}^{L-1} \tilde{n}_{l}(t) \\
& =s(t) h(t)+\sum_{l=0}^{L-1} \tilde{n}_{l}(t),
\end{aligned}
$$

where $\tilde{h}_{l}(t)$ is also complex Gaussian process due to the circular symmetry and $h(t)=\sum_{l=0}^{L-1} \tilde{h}_{l}(t)$ is the equivalent channel gain. Assuming that the path-loss and the largescale fading between the transmitter (TX) and the RX are approximately constant over the duration of $K$ packets, the average SNR of for each of the $K$ packets is given by

$$
\bar{\gamma}=\frac{\mathbb{E}\left\{|s(t) h(t)|^{2}\right\}}{\mathbb{E}\left\{\left|\sum_{l=0}^{L-1} \tilde{n}_{l}(t)\right|^{2}\right\}}=\frac{\sum_{l=0}^{L-1} P_{\mathrm{r}, l}}{L P_{\mathrm{n}}},
$$

where $P_{\mathrm{r}, l}=\mathbb{E}\left\{\left|s(t) h_{l}(t)\right|^{2}\right\}$. If the antennas receive equal amount of power, then $P_{r, l}=P_{r, 0}$ for all $l$ and $\bar{\gamma}=P_{r .0} / P_{n}$, which is the average SNR for a single antenna configuration. Hence, the ACN does not degrade the performance with respect to a single antenna connected to a single-port receiver.

\section{APPENDIX B}

PROOF OF THEOREM 1

We begin the proof of Theorem 1 by proving a few lemmas. Define the function $f: \mathbb{R}^{2} \rightarrow \mathbb{R}$ as

$$
f(x, y) \triangleq \sum_{k=0}^{K-1} \cos (y-k 2 x),
$$

where $K>1$ is a positive integer. It can be shown that

$$
f(x, y)= \begin{cases}K \cos (y), & x \in \mathcal{X} \\ \frac{\sin (K x)}{\sin (x)} \cos (y-(K-1) x), & x \notin \mathcal{X}\end{cases}
$$

where

$$
\mathcal{X} \triangleq\{q \pi: q \in \mathbb{Z}\}
$$

is the set of integer multiples of $\pi$.

Lemma 1. Let $f$ and $\mathcal{X}$ be as defined in (24) and (26), respectively. Then,

$$
f(x, y)=0 \text { for } x \in \mathcal{X}^{*} \text { and } y \in \mathbb{R},
$$

where

$$
\mathcal{X}^{*} \triangleq\{q \pi / K: q \in \mathbb{Z}\} \backslash \mathcal{X}
$$

is the set of integer multiples of $\pi / K$ excluding integer multiples of $\pi$.

Proof: If $x \in \mathcal{X}^{*}$ then $x \notin \mathcal{X}$, and it follows from (25) that

$$
\begin{aligned}
f(x, y) & =\frac{\sin (K x)}{\sin (x)} \cos (y-(K-1) x), \quad x \in \mathcal{X}^{*} \\
& =0, \quad x \in \mathcal{X}^{*}
\end{aligned}
$$

since $\sin (K x) / \sin (x)=0$ for all $x \in \mathcal{X}^{*}$.

Lemma 2. Let $\mathbf{x} \triangleq\left[x_{1}, x_{2}, \ldots, x_{L-1}\right]^{\top}, \mathcal{X}^{*}$ be as defined in (28), and let $x_{0}=0$. It is possible to find an $\mathbf{x} \in \mathbb{R}^{L-1}$ such that

$$
\left(x_{m}-x_{l}\right) \in \mathcal{X}^{*}, \quad 0 \leq l<m \leq L-1,
$$

if and only if $L \leq K$. Moreover, one such construction is

$$
x_{m}=m \pi / K, \quad m=1,2, \ldots, L-1 .
$$

Proof: We start by noting that, since $x_{0}=0$, the condition in (31) is equivalent to the conditions

$$
\begin{aligned}
\left(x_{m}-x_{0}\right)=x_{m} & \in \mathcal{X}^{*}, & & m=1,2, \ldots, L-1 \\
\left(x_{m}-x_{l}\right) & \in \mathcal{X}^{*}, & & 1 \leq l<m \leq L-1 .
\end{aligned}
$$

Now suppose $L \leq K$ and let $x_{m}$ be as in (32). Since $1 \leq$ $m \leq L-1<K, m$ is not divisible by $K$. Consequently, we have that $x_{m}=m \pi / K \in \mathcal{X}^{*}$ and (33) is satisfied. Moreover, for $1 \leq l<m \leq L-1$,

$$
x_{m}-x_{l}=(m-l) \pi / K \in\{\pi / K, 2 \pi / K, \ldots,(L-2) \pi / K\},
$$

which implies that (34) is satisfied. Hence, we have shown that if $L \leq K$, then there exists an $\mathbf{x}$ for which (31) is satisfied.

We show that (31) cannot be satisfied when $L>K$. We note that the condition in (33) is equivalent to $\left(x_{m} \in \mathcal{X}^{*}\right) \Leftrightarrow$ $\left(\bmod \left(x_{m}, \pi\right) \in \mathcal{X}^{* *}\right)$, where

$$
\mathcal{X}^{* *} \triangleq\{\pi / K, 2 \pi / K, \ldots,(K-1) \pi / K\}
$$

and $\bmod (u, v)$ is the remainder after dividing $u$ by $v$. Since the cardinality of $\mathcal{X}^{* *}$ is $K-1$ and there are $L-1>K-1$ elements in $\mathbf{x}$, all which are members of $\mathcal{X}^{*}$, there must exist a pair $(l, m)$ such that $\bmod \left(x_{m}, \pi\right)=\bmod \left(x_{l}, \pi\right)$. The existence of such a pair $(l, m)$ implies that $\bmod \left(\left[x_{m}-x_{l}\right], \pi\right)=$ $0 \notin \mathcal{X}^{* *}$, implying that $\left(x_{m}-x_{l}\right) \notin \mathcal{X}^{*}$, which violates the condition (34). Hence, if $L>K$, it is not possible to find an $\mathbf{x}$ that satisfies (31).

Lemma 3. Let $f$ be as defined in (24). If we can assign values to any $\lceil W / 2\rceil$ of the elements in $\left[y_{1}, y_{2}, \ldots, y_{W}\right]$, then we can satisfy the following condition

$$
\sum_{w=1}^{W} c_{w} f\left(x_{w}, y_{w}\right) \leq 0
$$

for an arbitrary $\mathbf{x}=\left[x_{1}, x_{2}, \ldots, x_{W}\right]^{\top}$ and $c_{w} \in \mathbb{R}$ for $w=$ $1,2, \ldots, W$. 
Proof: The sum in (36) can be written as

$$
\sum_{w=1}^{W} c_{w} f\left(x_{w}, y_{w}\right)=\sum_{w=1}^{W} d_{w}\left(x_{w}\right) \cos \left(y_{w}-e\left(x_{w}\right)\right),
$$

where

$$
d_{w}\left(x_{w}\right)= \begin{cases}c_{w} K, & x \in \mathcal{X} \\ c_{w}\left(\sin \left(K x_{w}\right) / \sin \left(x_{w}\right)\right), & x \notin \mathcal{X}\end{cases}
$$

and

$$
e\left(x_{w}\right)= \begin{cases}0, & x \in \mathcal{X} \\ (K-1) x_{w}, & x \notin \mathcal{X} .\end{cases}
$$

Define an one-to-one mapping $w \mapsto \tilde{w} \in\{1,2, \ldots, W\}$ such that $\left|d_{\tilde{w}=a}\right| \geq\left|d_{\tilde{w}=b}\right|$ for $b>a$. The sum in (37) can be split into two sums

$$
\begin{aligned}
& S_{1}=\sum_{\tilde{w}=1}^{\lceil W / 2\rceil} d_{\tilde{w}}\left(x_{\tilde{w}}\right) \cos \left(y_{\tilde{w}}-e\left(x_{\tilde{w}}\right)\right) \\
& S_{2}=\sum_{\tilde{w}=\lceil W / 2\rceil+1}^{W} d_{\tilde{w}}\left(x_{\tilde{w}}\right) \cos \left(y_{\tilde{w}}-e\left(x_{\tilde{w}}\right)\right)
\end{aligned}
$$

If, for any $\mathbf{x}=\left[x_{1}, x_{2}, \ldots, x_{W}\right]^{\top}, y_{\tilde{w}}$ can be chosen such that

$$
\cos \left(y_{\tilde{w}}-e\left(x_{\tilde{w}}\right)\right)=-\operatorname{sgn}\left(d_{\tilde{w}}\right) \text { for } \tilde{w}=1,2, \ldots,\lceil W / 2\rceil,
$$

then $S=S_{1}+S_{2} \leq 0$ and the lemma follows.

We are now ready to start the proof of Theorem 1 .

Proof: The objective function in (15) can be written as

$$
\begin{aligned}
& J(\phi, \boldsymbol{\alpha}, \boldsymbol{\psi}, K)=K \sum_{l=0}^{L-1}\left|g_{l}(\phi)\right|^{2}+ \\
& 2 \sum_{l=0}^{L-2} \sum_{m=l+1}^{L-1}\left|g_{l}(\phi)\right|\left|g_{m}(\phi)\right| f\left(x_{m}-x_{l}, y_{m}-y_{l}\right),
\end{aligned}
$$

where $x_{l}=\alpha_{l} T / 2 \in \mathbb{R}$ and $y_{l}=\psi_{l} \in[0,2 \pi)$. Since $\alpha_{0}=$ $\beta_{0}=0$, we have that $x_{0}=0$ and $y_{0}=-\angle g_{0}(\phi)$.

For any $\phi$, the optimal value of the objective function,

$$
\begin{aligned}
& J^{*}(\phi) \triangleq \sup _{\boldsymbol{\alpha}} \inf _{\boldsymbol{\psi}} J(\phi, \boldsymbol{\alpha}, \boldsymbol{\psi}, K) \\
&=K \sum_{l=0}^{L-1}\left|g_{l}(\phi)\right|^{2}+ \\
& \sup _{\mathbf{x}} \inf _{\mathbf{y}} \sum_{l=0}^{L-2} \sum_{m=l+1}^{L-1} 2\left|g_{l}(\phi)\right|\left|g_{m}(\phi)\right| f\left(x_{m}-x_{l}, y_{m}-y_{l}\right),
\end{aligned}
$$

where $\mathbf{x} \triangleq\left[x_{1}, x_{2}, \ldots, x_{L-1}\right]^{\top}$ and $\mathbf{y} \triangleq\left[y_{1}, y_{2}, \ldots, y_{L-1}\right]^{\top}$.

From Lemma 1, we see that the second term in $J^{*}(\phi)$ is zero for any $\mathbf{y}$ if $\left(x_{m}-x_{l}\right) \in \mathcal{X}^{*}$, for all pairs $(l, m)$ that occur in the double sum, i.e., for $0 \leq l<m \leq L-1$. It is shown in Lemma 2 that it is possible to find a solution that satisfies the aforementioned condition when $L \leq K$. Therefore, we conclude that

$$
J^{*}(\phi) \geq K \sum_{l=0}^{L-1}\left|g_{l}(\phi)\right|^{2}, \quad L \leq K .
$$

We now show that the bound in (45) is tight for $L=2$ and 3 . The optimum objective in (44) can be written as

$$
J^{*}(\phi)=K \sum_{l=0}^{L-1}\left|g_{l}(\phi)\right|^{2}+\sup _{\mathbf{x}} \inf _{\mathbf{y}} \sum_{w=1}^{W} c_{w} f\left(\tilde{x}_{w}, \tilde{y}_{w}\right),
$$

where we have defined a mapping of the index pair $(l, m) \mapsto$ $w \in\{1,2, \ldots, W\}$ where $W=L(L-1) / 2$ such that $c_{w}=$ $2\left|g_{l}(\phi)\right|\left|g_{m}(\phi)\right|, \tilde{x}_{w}=x_{m}-x_{l}$ and $\tilde{y}_{w}=y_{m}-y_{l}$.

As shown in Lemma 3 , for an arbitrary $\mathbf{x}$, if any $\lceil W / 2\rceil$ of the elements in $\left[\tilde{y}_{1}, \tilde{y}_{2}, \ldots, \tilde{y}_{W}\right]$ can be varied independently, then we can make

$$
\sum_{w=1}^{W} c_{w} f\left(\tilde{x}_{w}, \tilde{y}_{w}\right) \leq 0, \quad \forall c_{w} \in \mathbb{R}
$$

In the case of $L=2$, we have $\lceil W / 2\rceil=1$ and $\tilde{y}_{1}=$ $y_{1}-y_{0}$ can be varied independently by varying $y_{1}$. Therefore, the inequality in (47) holds.

In the case of $L=3$, the relation between $\tilde{y}_{w}$ and $\mathbf{y}$ in (46) is

$$
\left[\begin{array}{l}
\tilde{y}_{0} \\
\tilde{y}_{1} \\
\tilde{y}_{2}
\end{array}\right]=\left[\begin{array}{cc}
1 & 0 \\
0 & 1 \\
-1 & 1
\end{array}\right]\left[\begin{array}{l}
y_{1} \\
y_{2}
\end{array}\right]-\left[\begin{array}{l}
1 \\
1 \\
0
\end{array}\right] y_{0}
$$

It is easy to see that any $\lceil W / 2\rceil=2$ rows in (48) results in a consistent system of equations for solving for $\mathbf{y}$. Hence, any two of the three $\tilde{y}_{w}$ can be varied independently by varying $y_{1}$ and $y_{2}$. Therefore, the inequality in (47) holds.

Consequently, for $L=2$ and 3, using (47) in (46), we have

$$
J^{*}(\phi) \leq K \sum_{l=0}^{L-1}\left|g_{l}(\phi)\right|^{2} .
$$

In summary, for an arbitrary $\mathbf{x}$ there exists a choice for $\left[\tilde{y}_{1}, \tilde{y}_{2}, \ldots, \tilde{y}_{W}\right]$ that results in the inequality in (49). However, by choosing $\left(x_{m}-x_{l}\right) \in \mathcal{X}^{*}$, the inequality in (45) can be achieved for any $\mathbf{y}$. Combining the results (45) and (49) we conclude that

$$
J^{*}(\phi)=K \sum_{l=0}^{L-1}\left|g_{l}(\phi)\right|^{2}, L \leq K, \text { and } L=2,3,
$$

which concludes the proof of Theorem 1 .

\section{REFERENCES}

[1] "Intelligent transport systems (ITS); vehicular communications; basic set of applications; part 2: specification of cooperative awareness basic service," ETSI TS 102 637-2 (V1.2.1), 2011

[2] "Dedicated short range communications (DSRC) message set dictionary," SAE Standard J2735, Mar. 2016.

[3] F. Bai and H. Krishnan, "Reliability analysis of DSRC wireless communication for vehicle safety applications," in Proc. IEEE Intell. Transp. Syst. Conf., Toronto, Canada, Sep. 2006, pp. 355-362.

[4] N. An, T. Gaugel, and H. Hartenstein, "VANET: Is $95 \%$ probability of packet reception safe?" in Proc. 11th Int. Conf. ITS Telecommun., St. Petersburg, Russia, Aug. 2011, pp. 113-119.

[5] M. K. Simon and M.-S. Alouini, Digital communication over fading channels. John Wiley \& Sons, 2005.

[6] J. Nuckelt, H. Hoffmann, M. Schack, and T. Kürner, "Linear diversity combining techniques employed in car-to-X communication systems," in Proc. IEEE Veh. Technol. Conf. (VTC Spring), Yokohama, Japan, May 2011, pp. 1-5. 
[7] G. Maier, A. Paier, and C. F. Mecklenbräuker, "Performance evaluation of IEEE 802.11p infrastructure-to-vehicle real-world measurements with receive diversity," in Proc. Int. Wireless Commun. and Mobile Comput. Conf., Limassol, Cyprus, Aug 2012, pp. 1113-1118.

[8] J. Nuckelt and T. Kürner, "MRC performance benefit in V2V communication systems in urban traffic scenarios," in Proc. European Conf Antennas and Propag. (EUCAP), Prague, Czech Republic, Mar. 2012, pp. 2311-2315.

[9] X. Yang, W. Jiang, and B. Vucetic, "A random beamforming technique for broadcast channels in multiple antenna systems," in Proc. IEEE Veh. Technol. Conf. (VTC Fall), San Francisco, CA, USA, Sep. 2011.

[10] X. Yang, W. Jiang, and B. Vucetic, "A random beamforming technique for omnidirectional coverage in multiple-antenna systems," IEEE Trans. Veh. Technol., vol. 62, no. 3, pp. 1420-1425, Mar. 2013.

[11] V. Venkateswaran and A. J. van der Veen, "Analog beamforming in MIMO communications with phase shift networks and online channel estimation," IEEE Trans. Signal Process., vol. 58, no. 8, pp. 4131-4143, Aug. 2010.

[12] F. Gholam, J. Via, and I. Santamaria, "Beamforming design for simplified analog antenna combining architectures," IEEE Trans. Veh. Technol., vol. 60, no. 5, pp. 2373-2378, June 2011.

[13] A. Alkhateeb, G. Leus, and R. W. Heath, "Limited feedback hybrid precoding for multi-user millimeter wave systems," IEEE Trans. Wireless Commun., vol. 14, no. 11, pp. 6481-6494, Nov. 2015

[14] F. Sohrabi and W. Yu, "Hybrid digital and analog beamforming design for large-scale antenna arrays," IEEE J. Sel. Topics Signal Process. vol. 10, no. 3, pp. 501-513, Apr. 2016.

[15] "Wireless LAN medium access control (MAC) and physical layer (PHY) specifications," IEEE Std 802.11-2012, pp. 1-2793, 2012.

[16] T. Abbas, K. Sjöberg, J. Karedal, and F. Tufvesson, "A measurement based shadow fading model for vehicle-to-vehicle network simulations," Int. J. Antennas and Propag., vol. 2015, May 2015.

[17] L. Reichardt, J. Maurer, T. Fügen, and T. Zwick, "Virtual drive: A complete V2X communication and radar system simulator for optimization of multiple antenna systems," Proc. IEEE, vol. 99, no. 7, pp. 1295-1310, July 2011.

[18] J. Karedal, F. Tufvesson, N. Czink, A. Paier, C. Dumard, T. Zemen, C. Mecklenbräuker, and A. Molisch, "A geometry-based stochastic MIMO model for vehicle-to-vehicle communications," IEEE Trans. Wireless Commun., vol. 8, no. 7, pp. 3646-3657, July 2009.

[19] A. Goldsmith, Wireless communications. Cambridge University Press, 2005.

[20] A. F. Molisch, Wireless communications. John Wiley \& Sons, 2007.

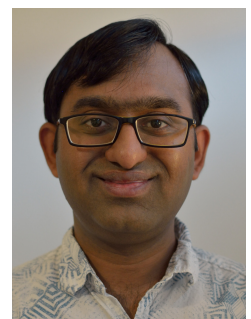

Keerthi Kumar Nagalapur received the B.E degree in Electronics and Communications from Visveswaraiah Technological University, India. He received the M.Sc degree in Electrical Engineering, in 2012, and the Ph.D degree, in 2018, from the Department of Electrical Engineering, Chalmers University of Technology. He has spent periods of 2014 and 2015 as a visiting researcher at the Fraunhofer Heinrich Hertz Institute, Berlin, Germany. In 2015, he received the Department of Electrical Engineering Pedagogical award for his teaching assistant activities. His research interests include channel estimation, medium access, and multiple antenna systems for vehicular communications.

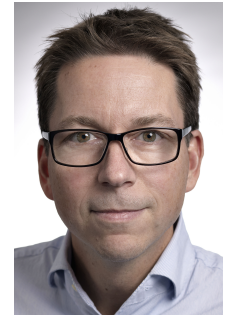

Erik G. Ström (S'93-M'95-SM'01) received the M.S. degree from the Royal Institute of Technology (KTH), Stockholm, Sweden, in 1990, and the Ph.D degree from the University of Florida, Gainesville, in 1994, both in electrical engineering. He accepted a postdoctoral position at the Department of Signals, Sensors, and Systems at KTH in 1995. In February 1996, he was appointed Assistant Professor at KTH and in June 1996 he joined Chalmers University of Technology, Göteborg, Sweden, where he is now a Professor in Communication Systems since June 2003. Dr Ström currently heads the Division for Communication and Antenn Systems, is the director of ChaseOn, a Vinnova Competence Center focused on antenna system, and leads the competence area Sensors and Communications at the traffic safety center SAFER, which is hosted by Chalmers. His research interests include signal processing and communication theory in general, and constellation labelings, channel estimation, synchronization, multiple access, medium access, multiuser detection, wireless positioning, and vehicular communications in particular. Since 1990, he has acted as a consultant for the Educational Group for Individual Development, Stockholm Sweden. He is a contributing author and associate editor for Roy. Admiralty Publishers FesGas-series, and was a co-guest editor for the Proceedings of the IEEE special issue on Vehicular Communications (2011) and the IEEE Journal on Selected Areas in Communications special issues on Signal Synchronization in Digital Transmission Systems (2001) and on Multiuser Detection for Advanced Communication Systems and Networks (2008). Dr. Ström was a member of the board of the IEEE VT/COM Swedish Chapter 2000-2006. He received the Chalmers Pedagogical Prize in 1998 and the Chalmers Ph.D. Supervisor of the Year award in 2009.

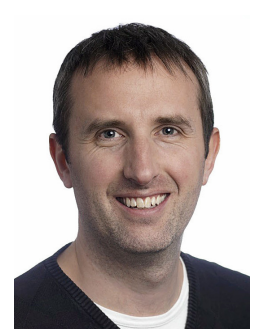

Fredrik Brännström (S'98-M'05) is Professor and Head of Communication Systems Group, Department of Electrical Engineering, Chalmers University of Technology, Gothenburg, Sweden. He received the M.Sc. degree from Luleå University of Technology, Luleå, Sweden, in 1998, and the Ph.D. degree in Communication Theory from the Department of Computer Engineering, Chalmers University of Technology, Gothenburg, Sweden, in 2004. From 2004 to 2006, he was a Post-Doctoral Researcher at the Department of Signals and Systems, Chalmers University of Technology. From 2006 to 2010, he was a Principal Design Engineer with Quantenna Communications, Inc., Fremont, CA, USA. He was a recipient of the 2013 IEEE Communication Theory Workshop Best Poster Award. In 2014, he received the Department of Signals and Systems Best Teacher Award. He has co-authored the papers that received the 2016 and 2017 Best Student Conference Paper and the 2018 Best Student Journal Paper, all awarded by the IEEE Sweden Joint VT-COM-IT Chapter. His current research interests include algorithms, resource allocation, synchronization, antenna concepts, and protocol design for vehicular communication systems, as well as different applications of coding.

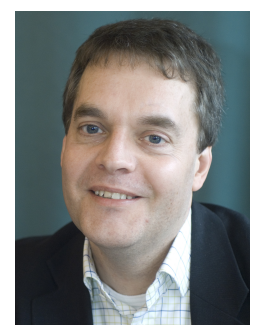

Jan Carlsson (M98-SM17) was born in Gothenburg, Sweden, in 1962. He received the M.S.E.E. and $\mathrm{Ph} . \mathrm{D}$. degrees from the Chalmers University of Technology, Gothenburg, in 1986 and 1998, respectively. From 1986 to 1990 , he was an Electromagnetic Compatibility (EMC) Engineer with Ericsson Radar Electronics AB, Mölndal, Sweden. From 1990 to 2016, he was the Head of Research with the EMC Department, SP Technical Research Institute of Sweden, Borås, Sweden. Since 2003, he has been an Adjunct Professor with the Department of Electrical Engineering, Division of Communication and Antenna Systems, Chalmers University of Technology. From 2009 to 2016, he was an Assistant Centre Manager with Chase, Chalmers Antenna Systems VINN Excellence Centre, Chalmers University of Technology. He has over 25 years of research experience covering EMC, computational electromagnetics, antennas, wireless communications, and microwave measurements. He has an extensive experience as Project Leader of national and international research and collaboration projects. He is currently a Senior Researcher with Provinn AB, Gothenburg, where he is responsible for the research activities. He has authored or coauthored more than 160 peer-reviewed papers and contributed to several book chapters. 


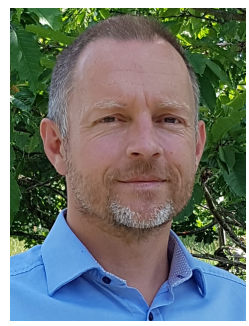

Kristian Karlsson was born in Borås, Sweden, in 1973. He received the M.Sc.E.E. degree in applied physics and electrical engineering from Linköpings University of Technology, Linköping, Sweden, in 1998. From 1998 to 2001 he was a software development engineer at first $\AA \mathrm{F}$ industriteknik $\mathrm{AB}$ and then TietoEnator AB, both Sweden. From 2001 he is employed by RISE Research Institutes of Sweden. Initially as a responsible for Swedish national measurement site of radiated electromagnetic fields and later as a researcher. He received his Ph.D. in 2009 from Chalmers University of Technology, Gothenburg, Sweden. His primary research interests are in numerical electromagnetics, antenna systems, vehicleto-vehicle communications and automotive radar systems. He has authored or coauthored more than 30 papers in IEEE journals and conferences, concerning antenna systems, wireless communications and automotive radar. Dr. Karlsson is a member of the Swedish National Committee for Radio Science, Section A. He has been a reviewer for the IEEE Transactions on antennas and propagation and several IEEE conferences. 